$$
\text { Pontifícia Universidade Católica }
$$

Marcelo Alfredo de Assis Fayal

Previsão de Vazão por Redes Neurais Artificiais e Transformada Wavelet

Dissertação de Mestrado

Dissertação apresentada como requisito parcial para obtenção do grau de Mestre pelo Programa de Pósgraduação em Engenharia Elétrica do Departamento de Engenharia Elétrica da PUC-Rio.

Orientadora: Profa. Marley Maria Bernardes Rebuzzi Vellasco Co-Orientador: Prof. Carlos Roberto Hall Barbosa

Rio de Janeiro 


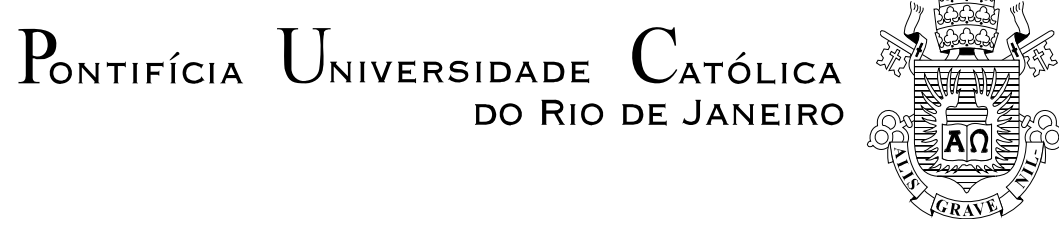

Marcelo Alfredo de Assis Fayal

\section{Previsão de Vazão por Redes Neurais Artificiais e Transformada Wavelet}

Dissertação apresentada como requisito parcial para obtenção do grau de Mestre pelo Programa de PósGraduação em Engenharia Elétrica do Departamento de Engenharia Elétrica do Centro Técnico Científico da PUCRio. Aprovada pela Comissão Examinadora abaixo assinada.

Dra. Marley Maria Bernardes Rebuzzi Vellasco Orientadora

Departamento de Engenharia Elétrica - PUC-Rio

Dra. Carlos Roberto Hall Barbosa

Co-Orientador

Departamento de Metrologia - PUC-Rio

Dr. Marco Aurélio Cavalcanti Pacheco Departamanto de Engenharia Elétrica - PUC-Rio

Dra. Karla Tereza Figueiredo Leite

UERJ

Prof. Gerson Zaverucha

COPPE/UFRJ

Prof. José Eugênio Leal Coordenador Setorial do Centro

Técnico Científico - PUC-Rio

Rio de Janeiro, 28 de fevereiro de 2008 
Todos os direitos reservados. É proibida a reprodução total ou parcial do trabalho sem autorização da universidade, do autor e do orientador.

\section{Marcelo Alfredo de Assis Fayal}

Graduou-se em Engenharia Elétrica no IME (Instituto Militar de Engenharia) em 1995, e especializou-se em Sistemas Computacionais na CCE/PUC-Rio, em 2004. Com foco na modelagem de novos sistemas de apoio a decisão, atua como integrante da Seção de Inteligência Tecnológica e da Coordenadoria de Pesquisa e Desenvolvimento de Projetos do CTEx (Centro Tecnológico do Exército).

Ficha Catalográfica

Fayal, Marcelo Alfredo de Assis

Previsão de vazão por redes neurais artificiais e transformada Wavelet / Marcelo Alfredo de Assis Fayal ; orientador: Marley Maria Bernardes Rebuzzi Vellasco ; co-orientador: Carlos Roberto Hall Barbosa. - 2008.

158 f. ; $30 \mathrm{~cm}$

Dissertação (Mestrado em Engenharia Elétrica) Pontifícia Universidade Católica do Rio de Janeiro, Rio de Janeiro, 2008.

Inclui bibliografia

1. Engenharia elétrica - Teses. 2. Previsão de vazão. 3. Redes neurais artificiais. 4. Transformada wavelet. I. Vellasco, Marley Maria Bernardes Rebuzzi. II. Barbosa, Carlos Roberto Hall. III. Pontifícia Universidade Católica do Rio de Janeiro. Departamento de Engenharia Elétrica. IV. Título. 
Dedico este trabalho a Maria das Graças, Mariana Banana Paçoca Estrelinha e a Maria Eduarda Ligeirinha. 


\section{Agradecimentos}

De início, por questão de justiça, agradeço aos meus orientadores Marley e Hall, pelas centenas de horas reservadas para a orientação deste trabalho. Pela enorme paciência, confiança e pela extrema cordialidade com que sempre fui tratado por eles. Sinto-me um afortunado.

Agradeço ao pessoal do ICA, em especial a Karla Figueiredo e a Iury Steiner.

Agradeço o apoio imprescindível prestado pela equipe de engenheiros da Intratec Consulting, em especial, à figura de seu diretor Luiz Felipe de Souza Tavares e de seus engenheiros Jansley Pascoal, Ademir Marreiros, Daniel Barros e Thiago Carneiro, sem o qual seria impossível a realização deste trabalho.

Agradeço ao amigo Adrian Pizzinga, jovem doutor e mago da estatística, por suas inúmeras colaborações desde o começo deste trabalho.

Agradeço ao meu pai e ao meu irmão pelo exemplo de bondade, carinho e honestidade. Pelo amparo nas horas difíceis, pelo sentido de que na vida é preciso perseverar sempre e sobretudo por terem sido os exemplos através dos quais sempre me guiei.

Agradeço a minha mãe por todos os momentos puros, felizes e de tranqüilidade da minha infância, juventude e maturidade, passando paz interior e apaziguando o coração e serenando as angústias das pessoas à sua volta.

Agradeço a Senhora Nilca, por sua bondade e sobretudo por amar minhas filhas.

Agradeço, ainda, aos meus amigos, mentores, e oficiais exemplares, os Senhores: Coronel Maurílio, Coronel Josedes e Coronel Ângelo.

Finalmente, agradeço a Deus, todos os santos, arcanjos e anjos do céu pela saúde das minhas duas filhas Mariana e Maria Eduarda. 


\section{Resumo}

Fayal, Marcelo Alfredo de Assis; Vellasco, Marley Maria Bernardes Rebuzzi (Orientadora). Previsão de Vazão Por Redes Neurais Artificiais e Transformada Wavelet. Rio de Janeiro, 2008. 158p. Dissertação de Mestrado Departamento de Engenharia Elétrica, Pontifícia Universidade Católica do Rio de Janeiro.

O sistema hidroelétrico é responsável por $83,7 \%$ da energia elétrica gerada no país. Assim sendo, a geração de energia elétrica no Brasil depende basicamente das vazões naturais que afluem aos aproveitamentos hidroelétricos distribuídos por doze bacias hidrográficas no país. Sendo o Operador Nacional do Sistema Elétrico (ONS) o órgão responsável por elaborar a previsão e a geração de cenários de vazões naturais médias diárias, semanais e mensais para todos os locais de aproveitamentos hidroelétricos do Sistema Interligado Nacional (SIN), a qualidade da previsão da vazão natural é de suma importância para este órgão. A qualidade dessa previsão impacta diretamente no planejamento e em programas de operação do SIN, tal como o Programa Mensal de Operação - PMO. Mesmo com a melhoria na qualidade da previsão de vazões por meio da criação e adoção dos mais diversos modelos determinísticos e estocásticos nos últimos anos, os erros de previsão são, ainda, significativos. Deste modo, o objetivo principal desta dissertação foi propor um novo modelo capaz de proporcionar um significativo ganho de qualidade na previsão de vazões nas regiões dos aproveitamentos hidrelétricos das bacias hidrográficas do país. O modelo proposto, baseado em redes neurais, tem como ferramenta primordial a utilização de transformadas wavelets, que filtram os dados históricos de vazões, ou seja, as entradas das redes neurais de previsão, dividindo esses dados de entrada (sinais) em diversas escalas, no intuito de que as redes neurais possam melhor analisá-los. Para verificar a eficácia do modelo proposto, aqui denominado MIP (Modelo Inteligente de Previsão), procedeu-se um estudo de caso que realiza a previsão de vazões naturais incrementais médias diárias e semanais no trecho incremental entre as Usinas Hidroelétricas (UHE) Porto Primavera, Rosana e Itaipu da Bacia do Rio Paraná, chegando-se a um erro de aproximadamente $3,5 \%$ para previsão de vazões um dia à frente, $16 \%$ para 12 dias à frente, e $9 \%$ para previsão média semanal. Esta dissertação objetiva, também, investigar a eficácia do uso de informações das precipitações observadas e previstas na previsão de vazão, em conjunção com o uso do histórico de vazões.

\section{Palavras-chave}

Previsão de vazão; informações de precipitação; modelos chuva-vazão, redes neurais artificiais, transformada wavelet 


\section{Abstract}

Fayal, Marcelo Alfredo de Assis; Vellasco, Marley Maria Bernardes Rebuzzi (Advisor). Outflow Forecast Based on Artificial Neural Netorks and Wavelet Transform. Rio de Janeiro, 2008. 158p. MSc Dissertation - Departamento de Engenharia Elétrica, Pontifícia Universidade Católica do Rio de Janeiro.

The hydroelectricity system is responsible for $83.7 \%$ of the electric energy generated at Brazil. Therefore, the generation of electric power in Brazil depends basically on the natural flow rates distributed by twelve basins in the country. The quality of prediction of natural flow is of crucial importance for the Brazilian governmental agency, ONS (from the portuguese language Electrical National Operator System), responsible for preparing the forecast and the generation of scenarios of daily, weekly and monthly average natural streamflows of all places of hydroelectric exploitations of SIN (from the portuguese language National Linked System). The quality of that forecast impacts directly in the planning and operation programs of SIN, for example, the PMO (from the portuguese language Monthly Operation Program). Even with the improvement in the quality of river flow forecasts through the creation and adoption of the various deterministic and stochastic models in recent years, the errors of forecasting are still significant. Thus, the main goal of this dissertation was proposing a new model capable of providing a significant improvement in Streamflow forecasts in regions of exploitations of hydroelectric basins of the country. The proposed model, based on neural networks, has the primary tool the use of wavelet transforms, to filter streamflows historical data, or the entries of predict neural networks, dividing the input data (signals) in several scales, in order that the neural networks can better analyse them. In order to check the effectiveness of the proposed model, here called MIP (from the portuguese language Forecast Intelligent Model), it was developed a case study to forecast daily and weekly average of natural incremental streamflows between the Hydroelectric Plants: Porto Primavera, Rosana e Itaipu belonging to the the Parana River Basin. The model reaches up an error of about 3,5\% to estimates of streamflows one day ahead, $16 \%$ to 12 days ahead, and $9 \%$ for average weekly forecast. This thesis aims to also investigate the effectiveness of the use of information of observed and predicted rainfall in the forecast flow, in conjunction with the use of the historical streamflows.

\section{Keywords}

Outflow forecast, artificial neural networks, wavelet transform. 


\section{Sumário}

1. Introdução 14

$\begin{array}{ll}\text { 1.1. Motivação } & 14\end{array}$

1.1.1. Previsão Hidrológica no âmbito do SIN 15

1.1.2 Previsão Hidrológica usando Redes Neurais, chuvas observadas e

$\begin{array}{lr}1.2 \text {. Objetivos } & 18 \\ 1.3 . \text { Descriço do Trabalho } & 18\end{array}$

1.3. Descrição do Trabalho 19

1.4. Organização da Dissertação 21

2. Modelagem Hidrológica 23

2.1. Introdução 23

2.2. Histórico 25

2.3. Modelos atualmente usados no Mundo 28

2.4. Escolha do Modelo 38

3. Métodos e Modelos $\quad 40$

3.1. Método de Seleção de Variáveis 40

3.1.1. Introdução 40

3.1.2. Método do Estimador por Mínimos Quadrados (LSE) 41

3.1.2.1. Descrição 41

3.1.2.2. Algoritmo do LSE 42

3.1.2.3. Importância das Variáveis de Entrada 44

3.2. Redes Neurais Artificiais $\quad 44$

3.2.1. Histórico 44

3.2.2. Estrutura do Neurônio 46

$\begin{array}{ll}\text { 3.2.3. Estrutura da Rede } & 48\end{array}$

3.2.4. Processamento Neural 49

3.2.5. Aprendizagem e Treinamento $\quad 50$

3.3. Wavelets

3.3.1. Introdução

3.3.2. Perspectiva Histórica $\quad 56$

3.3.3. A Transformada de Fourier $\quad 58$

3.3.3.1. Análise de Fourier Short-Time (Windowed) 61

3.3.4. A Transformada Wavelet 63

3.3.4.1. A Transformada Wavelet Contínua (TWC) 65

3.3.4.2. A Transformada Wavelet Discreta (TWD) 69

3.3.4.3. O Algoritmo Piramidal de Mallat: Aproximações e Detalhes $\quad 70$

3.3.4.4. Decomposição e Múltiplos Níveis $\quad 72$

3.3.4.5. Reconstrução Wavelet $\quad 73$

3.3.4.6. Reconstrução de Aproximações e Detalhes 74

3.3.4.7. Famílias Wavelets $\quad 75$

4. Modelo Inteligente de Previsão (MIP) 78

4.1. Modelo Proposto $\quad 78$

4.2. Módulo 1 - Módulo de Tratamento dos Dados $\quad 80$

4.3. Módulo 2 - Módulo Wavelet $\quad 87$

4.4. Módulo 3 - Módulo de Redes Neurais 88 
5. Estudo de Caso 90

5.1. Introdução 90

5.2. Módulo 1 - Tratamento dos Dados 93

5.2.1. Séries Histórias Disponíveis 93

5.2.2. Cálculo da chuva de Thiessen para a Bacia e Completamento dos Dados 95

5.2.3. Confecção das Matrizes de Dados e Seleção de Variáveis 99

5.3. Previsão sem Transformação Wavelet 104

5.3.1. Características e Modelagem das Redes Neurais 104

5.3.2. Previsão de Vazões 106

5.3.3. Avaliação dos Resultados 111

5.4. Previsão com Transformação Wavelet 112

5.4.1. Características dos Módulos 2 e $3 \quad 112$

5.4.2. Testes Preliminares 112

5.5. Previsão sem Wavelet x Previsão com Wavelet 118

6. Conclusões e Trabalhos Futuros $\quad 120$

6.1. Conclusões 120

$\begin{array}{ll}\text { 6.2. Trabalhos Futuros } & 121\end{array}$

$\begin{array}{ll}\text { Referências Bibliográficas } & 123\end{array}$

$\begin{array}{lr}\text { Apêndice } 1 & 130\end{array}$

Apêndice 2 138

Apêndice $3 \quad 140$

Apêndice 4 142

Apêndice 5

Apêndice $6 \quad 145$

Apêndice $7 \quad 153$

Apêndice 8 155

Apêndice $9 \quad 158$ 


\section{Lista de figuras}

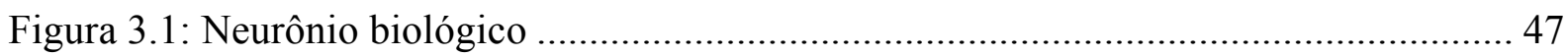

Figura 3.2: Descrição de um k-ésimo neurônio .................................................................. 47

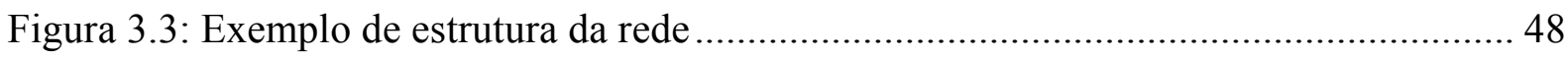

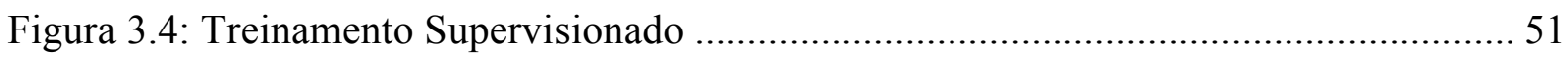

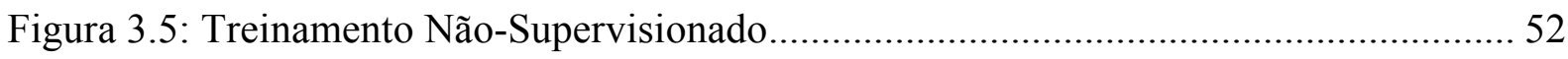

Figura 3.6: Conexões de um neurônio de camada escondida ............................................. 54

Figura 3.7: A função na parte de baixo é composta pelas três funções acima dela ............... 59

Figura 3.8: Dois gráficos ilustrativos da STFT, também conhecida como

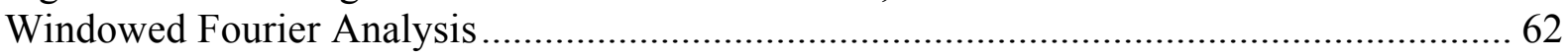

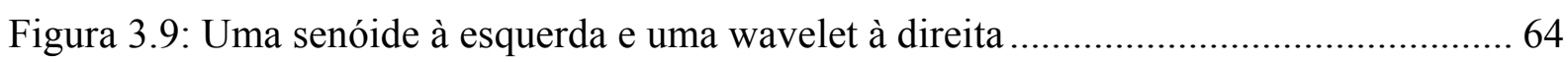

Figura 3.10: Produto de 2 seções de uma função $a$ pela wavelet $b$, gerando áreas $d$ e $f$ - cujas áreas são os coeficientes Wavelets ............................................................... 64

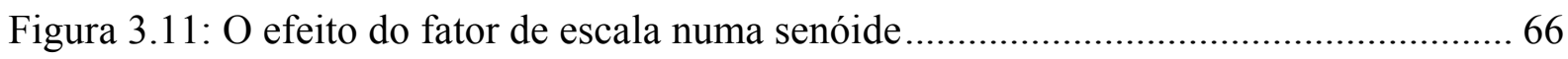

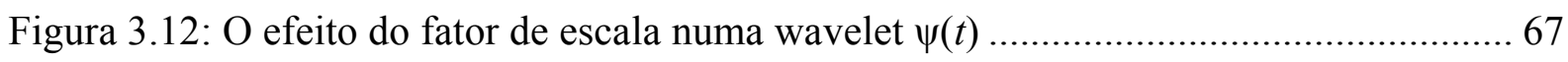

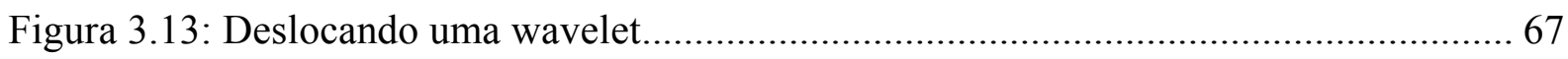

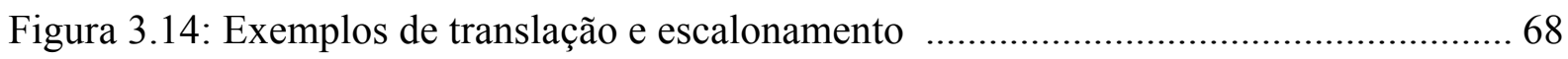

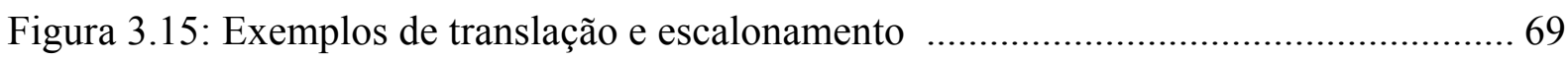

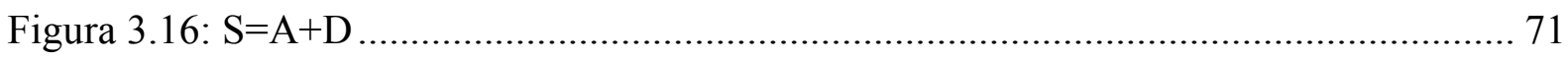

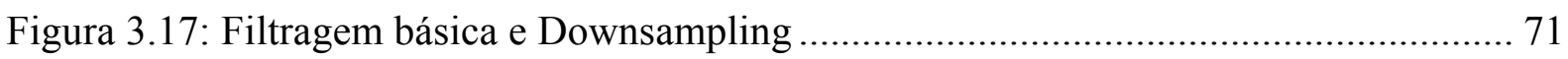

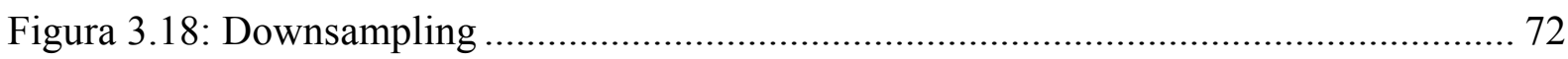

Figura 3.19: Árvore de decomposição wavelet .................................................................. 72

Figura 3.20: Detalhes dos coeficientes na árvore de decomposição wavelet........................ 73

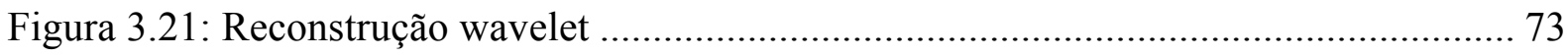

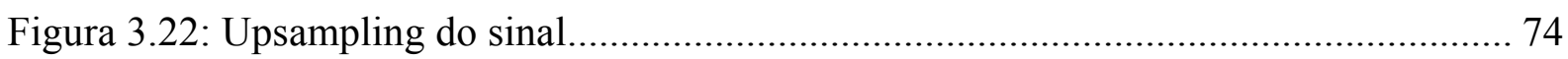

Figura 3.23: Construção dos detalhes e aproximações ...................................................... 74

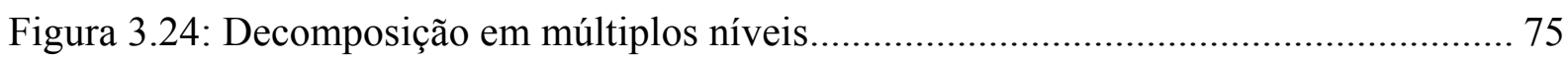

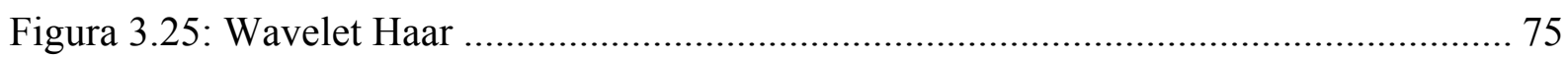

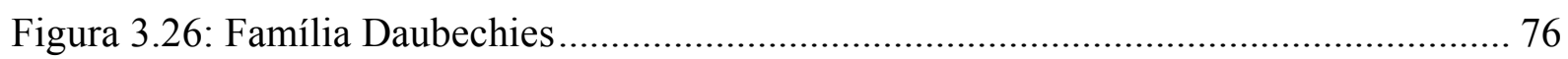

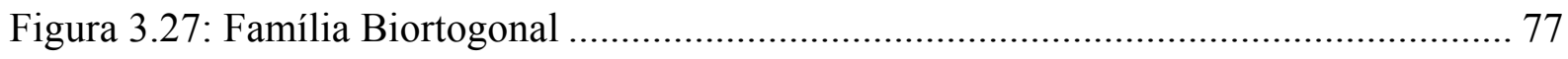

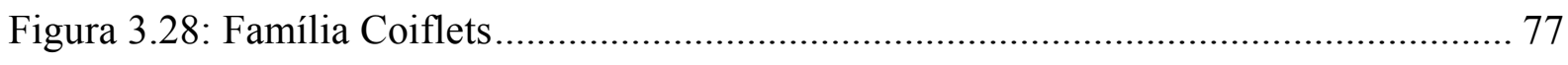

Figura 4.1: Modelo MIP composto pelos módulos 1,2 e 3 .................................................. 79

Figura 4.2: Previsão de vazão incremental média diária do $1^{\circ}$ ao $12^{\circ}$ dia ........................... 80 
Figura 4.3: Três previsões de vazões médias semanais ................................................. 80

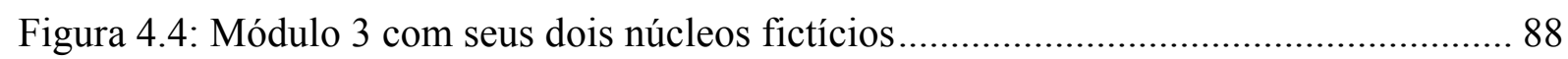

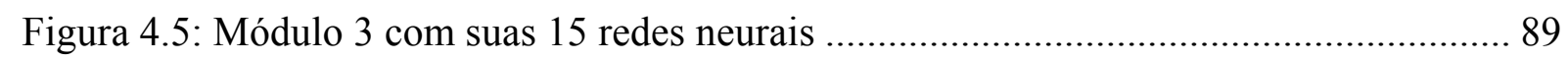

Figura 5.1: Mapa da bacia incremental de Itaipu e sub-bacias .......................................... 91

Figura 5.2: Aproveitamentos hidrelétricos onde aparecem as UHE Rosana, Porto

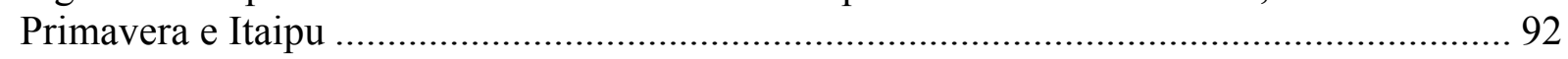

Figura 5.3: Localização dos 37 postos pluviométricos [88] ................................................ 94

Figura 5.4: Os 37 postos pluviométricos e suas relativas áreas de domínio [88] .................. 96

Figura 5.5: Pesos de Thiessen dos 37 postos pluviométricos ............................................. 97

Figura A.1.1: Componentes de um ciclo hidrológico ...................................................... 131

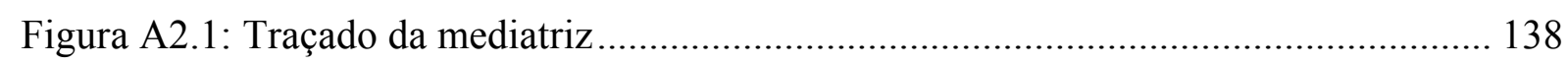

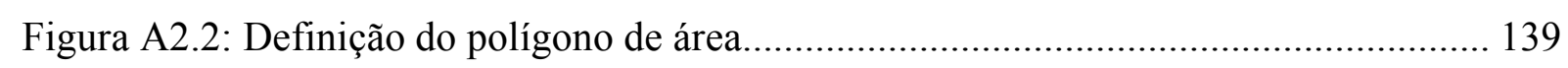

Figura A.5.1: Parada tardia e ponto ótimo de generalização .............................................. 143

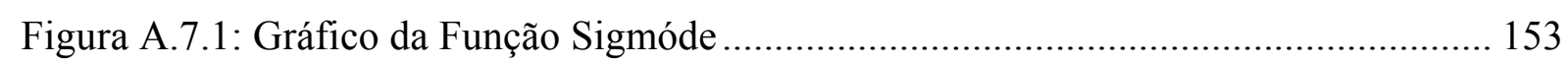

Figura A.7.2: Gráfico da Função Sigmóide com $a$ tendendo ao infinito ............................ 154

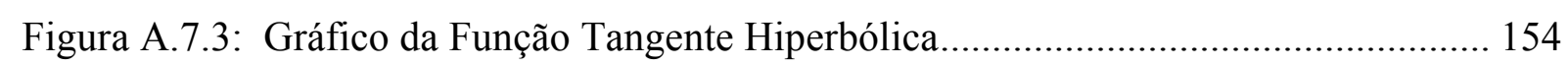

Figura A.8.1: Vazões observada e simulada de Vmd2 com Wavelet .................................. 155

Figura A.8.2: Vazões observada e simulada de Vmd2 sem Wavelet................................. 155

Figura A.8.3: Vazões observada e simulada de Vmd4 com Wavelet .................................. 156

Figura A.8.4: Vazões observada e simulada de Vmd4 sem wavelet .................................. 156

Figura A.8.5: Vazões observada e simulada de Vmd11 com wavelet ................................. 157

Figura A.8.6: Vazões observada e simulada de Vmd11 sem wavelet................................. 157 


\section{Lista de tabelas}

Tabela 1.1: Índices MAPE (\%) dos modelos de previsão de vazões PREVIVAZ

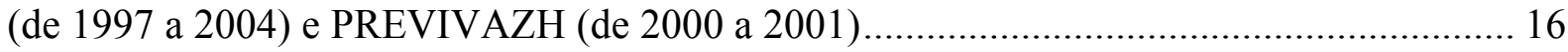

Tabela 1.2: Resultados dos modelos de previsão de vazões médias semanais ...................... 17

Tabela 1.3: Resultados dos modelos de previsão de vazões médias semanais NSRBN e PARMA

Tabela 2.1: Relação entre as diversas áreas apoiadas pela modelagem hidrológica e respectivos modelos.

Tabela 5.1: Postos pluviométricos e respectivos percentuais de falhas

Tabela 5.2: Tabela com os dados dos 5 postos fluviométricos da Bacia do Rio

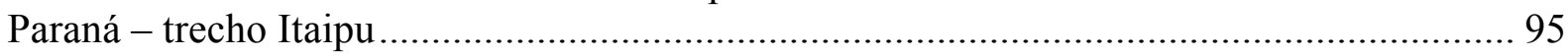

Tabela 5.3: Tabela de pesos de Thiessen relativos aos 37 postos pluviométricos ................ 96

Tabela 5.4: Ajuste da matriz de chuvas médias de Thiessen ............................................. 98

Tabela 5.5: Tabela de formação das matrizes de dados .................................................... 99

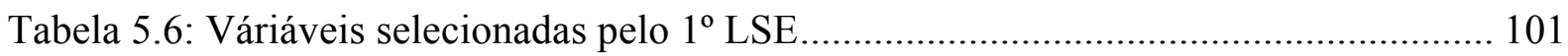

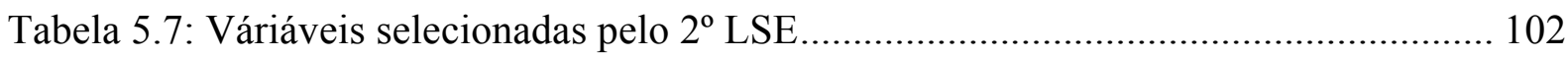

Tabela 5.8: Resultados das previsões semanais sem uso de Wavelets ............................... 107

Tabela 5.9: Resultados das previsões diárias sem uso de Wavelets .................................. 108

Tabela 5.10: Melhores resultados obtidos e respectivos conjuntos de entrada .................... 110

Tabela 5.11: Tabela comparativa dos resultados com e sem ETA .................................... 111

Tabela 5.12: Previsões usando wavelets daubechies ....................................................... 113

Tabela 5.13: Previsões usando como entradas janela de 3 valores anteriores da

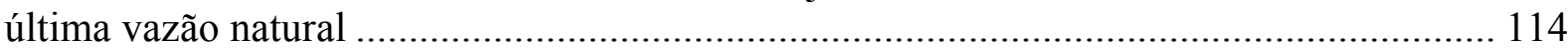

Tabela 5.14: Previsões usando wavelet coif 5 e como entradas 5 valores anteriores

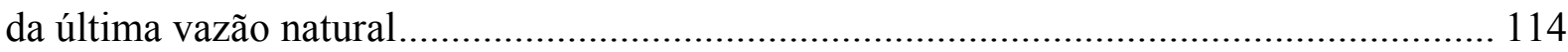

Tabela 5.15: Previsões usando wavelet coif 5 e bior e como entradas 7 valores anteriores da última vazão natural

Tabela 5.16: Previsões usando wavelet daubechies e como entradas 7 valores anteriores da última vazão natural

Tabela 5.17: Previsões usando wavelet $d_{25}$

Tabela 5.18: Seis Previsões usando wavelet $\mathrm{db}_{25}$

Tabela 5.19: Previsões diárias usando wavelet $d_{2}$ e 7 valores anteriores de vazões diárias

Tabela 5.20: Previsão sem wavelet x Previsão com wavelet

Tabela A.1.1: Características de modelos usados no gerenciamento de recursos hídricos 
Gráfico/Tabela A.6.1: Graus de importância em relação à Vms1 145

Gráfico/Tabela A.6.2: Graus de importância em relação à Vms2 145

Gráfico/Tabela A.6.3: Graus de importância em relação à Vms3 146

Gráfico/Tabela A.6.4: Graus de importância em relação à Vmd1 146

Gráfico/Tabela A.6.5: Graus de importância em relação à Vmd2 147

Gráfico/Tabela A.6.6: Graus de importância em relação à Vmd3 147

Gráfico/Tabela A.6.7: Graus de importância em relação à Vmd4 148

Gráfico/Tabela A.6.8: Graus de importância em relação à Vmd5 148

Gráfico/Tabela A.6.9: Graus de importância em relação à Vmd6 149

Gráfico/Tabela A.6.10: Graus de importância em relação à Vmd7 149

Gráfico/Tabela A.6.11: Graus de importância em relação à Vmd8 150

Gráfico/Tabela A.6.12: Graus de importância em relação à Vmd9 150

Gráfico/Tabela A.6.13: Graus de importância em relação à Vmd10 151

Gráfico/Tabela A.6.14: Graus de importância em relação à Vmd11 151

Gráfico/Tabela A.6.15: Graus de importância em relação à Vmd12 152 